\title{
Mitral valve closure and left ventricular filling time in patients with VDD pacemakers Assessment of the onset of left ventricular systole and the end of diastole
}

\author{
HELENE VON BIBRA, ALEXANDER WIRTZFELD, ROGER HALL, * \\ KURT ULM, HANS BLÖMER \\ From the First Medical Clinic of the Technical University, Munich, Federal Republic of Germany
}

SUMMARY The effect of mitral valve closure on left ventricular filling time and its relation to the onset of systole were assessed from mitral valve echocardiograms and simultaneous apex cardiograms in 21 normal subjects, 11 patients with left bundle branch block, and 19 patients with VDD pacemakers programmed for atrioventricular intervals of 50, 150, and $250 \mathrm{~ms}$. The interval between the electrocardiograph $Q$ wave and the apex cardiogram upstroke was similar in normal subjects and patients with left bundle branch block, but was significantly longer in patients with VDD pacemakers at all atrioventricular intervals. Similarly there was little difference in the time interval between the $Q$ wave and mitral valve closure in normal individuals and patients with left bundle branch block but this was considerably delayed in VDD pacemaker patients with the atrioventricular interval set at $50 \mathrm{~ms}$. With increasing atrioventricular intervals the mitral valve closed significantly earlier, whereas the onset of left ventricular systole and the timing of mitral valve opening remained unchanged. Thus as a result of earlier mitral valve closure left ventricular filling time decreased progressively as the atrioventricular interval was increased. Since the onset of left ventricular systole, with respect to left ventricular stimulation, is considerably delayed in VDD pacemaker patients a short atrioventricular interval is required in these patients to maintain the normal time relations between atrial and ventricular contraction and hence maximise left ventricular filling.

Left ventricular filling provides the blood volume for subsequent left ventricular ejection. One of its important determinants is left ventricular filling time, which is determined by the mitral valve movement. This injection time depends not only on cardiac cycle length but also on the mechanisms which time mitral valve closure. The timing of mitral valve closure can be altered by electrical delay of ventricular depolarisation ${ }^{1-3}$ and in atrioventricular conduction disturbances. ${ }^{4-6}$ These two conditions

Requests for reprints to Dr $\mathrm{H}$ von Bibra, I Medizinische Klinik rechts der Isar, Ismaninger Strasse 22, 8000 Munich 80, Federal Republic of Germany.

*The Royal Victoria Infirmary, Newcastle upon Tyne.

Accepted for publication 17 December 1985 coexist in patients with dual chamber pacemakers since ventricular depolarisation begins from an abnormal focus and is delayed and the PR interval can be altered by programming the pacemaker. Since the relative timing of atrial and ventricular contraction can be altered simply by reprogramming the pacemaker these patients offer a unique opportunity to study the effect of these events on mitral valve movements and left ventricular filling time.

This study was designed to examine noninvasively the relations between electrical and mechanical events in patients with VDD pacemakers over a wide range of atrioventricular intervals. The movements of the mitral valve and hence left ventricular filling time were detected by $M$ mode echocardiography as was aortic valve opening which times the onset of left ventricular ejection. The onset of mechanical systole was detected by the upstroke 
of an apex cardiogram and electrocardiogram. A group of normal controls was studied. Finally patients with left bundle branch block were also studied to examine the possible effects of conduction disturbance of a type similar to that seen in right ventricular pacing.

This information is of theoretical interest because of the insight that it gives into the mechanisms underlying mitral valve motion and particularly mitral valve closure. It is also of considerable practical importance. Patients with myocardial impairment who receive dual chamber pacemakers supposedly benefit from the improvement in left ventricular diastolic function produced by coordinating atrial and ventricular contraction. This study attempts to establish the atrioventricular intervals that provide the longest left ventricular filling time and thus should maximise the clinical benefits of this type of pacing.

\section{Patients and methods}

We studied 51 patients who had been informed about the nature of the study and had consented to take part in it. These patients were divided into three groups:

Control group-There were 21 controls (12 men, 9 women; age range 24-57 years). Cardiac disease was excluded in these subjects by a normal history, physical examination, electrocardiogram, and chest $x$ ray.

Left bundle branch block-There were 11 patients (seven men, four women; age range 39-70 years). They had normal PR intervals but left bundle branch block in association with the following diseases: hypertension (2), hypertrophic cardiomyopathy (3), congestive cardiomyopathy (3), ischaemic heart disease (1) and aortic valve replacement (2). This group was included so that we could investigate the effects of abnormal ventricular depolarisation of a type similar to that occurring in patients with right ventricular pacing.

Pacemaker group-There were 19 patients (11 men and eight women, age range 21-75 years) in this group. All showed the electrocardiographic pattern of left bundle branch block due to right ventricular stimulation by a dual chamber pacemaker. The pacemakers, all programmed in VDD mode, had been implanted for complete heart block (17 patients) or second degree heart block (2 patients) 3-18 months before the study. Ten of these patients had presented with symptoms of left ventricular failure (New York Heart Association grade II-III); three had ischaemic heart disease, and two had undergone aortic valve replacement. In the other nine patients without symptoms of left ventricular failure the aetiology of the heart block was not clear apart from one patient with congenital heart block and another with a history of myocarditis. All patients were studied with their VDD pacemakers programmed for the following atrioventricular intervals: 50, 150, and $250 \mathrm{~ms}$. The protocol began with an atrioventricular interval of $150 \mathrm{~ms}$, and this was followed by the two other intervals in random sequence. All measurements were made on beats in which ventricular pacing was triggered by a $P$ wave after the programmed delay.

INVESTIGATIONS

Echocardiograms to detect mitral and aortic valve movements were obtained from all patients by means of a Hewlett Packard 77020 A ultrasound system with a $3.5 \mathrm{MHz}$ transducer. $\mathrm{M}$ mode echocardiograms were recorded both at the level of the aortic valve and the mitral valve at a paper speed of $100 \mathrm{~mm} / \mathrm{s}$. Electrocardiograms were recorded simultaneously in all patients. A simultaneous apex cardiogram was recorded in 10 randomly selected patients in each group. The apex cardiogram was recorded because the onset of its upstroke coincides with the onset of left ventricular pressure rise. ${ }^{7}$ Thus mitral valve movements and electrocardiographic events could be related to the onset of mechanical systole in these subgroups. Each observation was the mean value of measurements taken from 10 consecutive cardiac cycles.

The following time intervals were measured from the preceding electrocardiographic pacemaker spike or $\mathrm{Q}$ wave as appropriate: $(a)$ the onset of the upstroke of the apex cardiogram; $(b)$ mitral valve closure (MC); (c) aortic valve opening (AO); (d) mitral valve opening (MO).

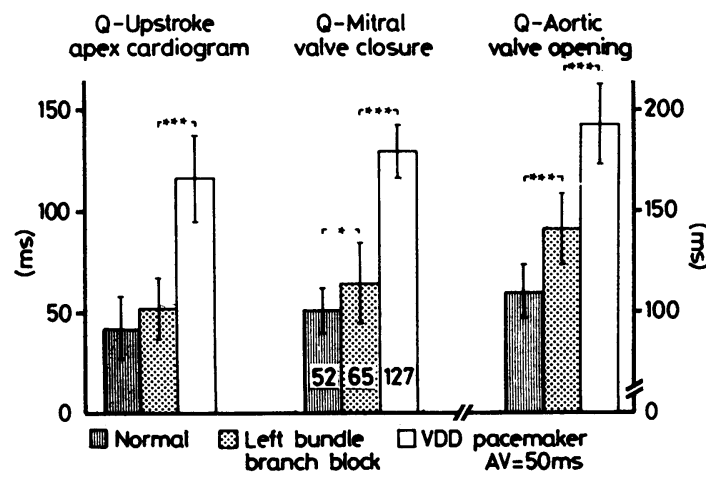

Fig. 1 Timing (mean (SD)) of upstroke of apex cardiogram, of mitral valve closure, and of aortic valve opening with reference to the $Q$ wave or the pacemaker spike in normal individuals, patients with left bundle branch block, and in patients with VDD pacemakers set at an atrioventricular interval of $50 \mathrm{~ms} .{ }^{\star} p<0.05,{ }^{\star}{ }^{\star} p<00.001$. 


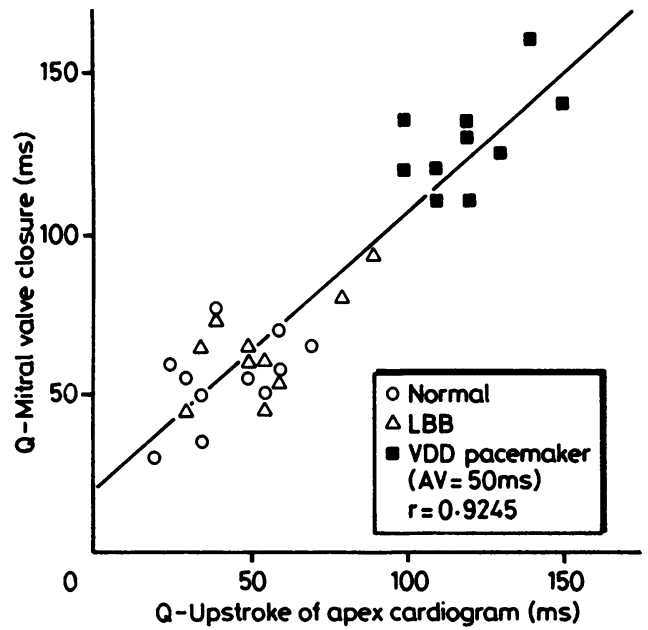

Fig. 2 Correlation between onset of left ventricular systole (taken as timing of the apex cardiographic upstroke) and end of preceding diastole (taken as mitral valve closure) arranged in groups according to the increasing delay of intraventricular conduction - that is normal individuals, patients with left bundle branch block ( $L B B$ ), and patients with VDD pacemakers set at an atrioventricular interval of $50 \mathrm{~ms}$.

The onset of mitral A wave (A) was timed from the succeeding pacemaker spike or $Q$ wave and the cycle length ( $R-R$ interval) was also recorded.

$\mathrm{LV}$ filling time (FT, ms) was calculated as follows:

$$
\mathrm{FT}=\mathrm{RR}-\mathrm{MO}+\mathrm{MC}
$$

and normalised by expressing it as a percentage of cycle length $(\% \mathrm{FT})$.

$$
\% \mathrm{FT}=\frac{\mathrm{FT}}{\mathrm{RR}} \times 100
$$

The statistical significance of differences between mean values in the three groups was analysed by the Kruskal Wallis test and by the Mann Witney test. Differences between mean values within the pacemaker group were analysed by the Friedman test and the Wilcoxon rank test. Correlation coefficients were calculated by the least squares method.

\section{Results}

Increasing delay of intraventricular depolarisation, its influence on the end of diastole and onset of systole

Figure 1 shows the intervals between the $Q$ wave or pacing spike and the onset of the upstroke of the apex cardiogram, mitral valve closure, and aortic valve opening. In this section the results given were obtained with pacemakers programmed to an atrioventricular interval of $50 \mathrm{~ms}$, from patients with left

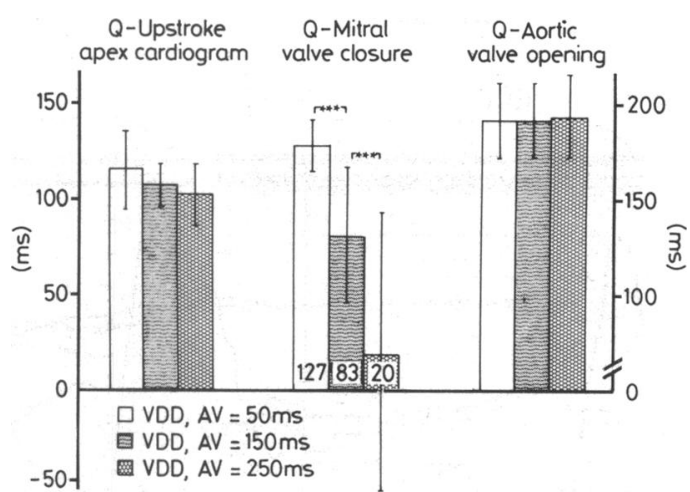

Fig. 3 Timing (mean (SD)) of upstroke of apex cardiogram, of mitral valve closure, and of aortic valve opening in VDD pacemaker patients at atrioventricular intervals of 50,150 , and $250 \mathrm{~ms} .{ }^{\star \star \star} \mathrm{p}<0.001$.

bundle branch block, and from normal controls. Mean cycle length was the same in all three groups of patients. The upstroke of the apex cardiogram occurred 44 (16) ms (mean (SD)) after the electrocardiographic $Q$ wave in normal subjects and was similarly delayed $(52(18) \mathrm{ms})$ in patients with left bundle branch block. It was considerably delayed in pacemaker patients, occurring 119 (21) ms $(p<0.001)$ after the pacing spike. Mitral valve closure was 52 (11) $\mathrm{ms}$ after the $Q$ wave in normal individuals and slightly later $(65(20) \mathrm{ms})$ in patients with bundle branch block $(p<0.05)$. It was markedly delayed in pacemaker patients being 127 (14) ms after the pacing spike $(p<0.001)$ compared with both other groups. Aortic valve opening demonstrated a similar pattern in the three groups. It occurred 111 (14) ms after the $Q$ wave in normal subjects, 143 (19) $\mathrm{ms}$ after the $Q$ wave in patients with left bundle branch block $(p<0.001)$, and 193 (20) $\mathrm{ms}$ after the pacing spike in pacemaker patients $(\mathrm{p}<0.001)$.

A positive linear correlation was demonstrated between the onset of left ventricular systole (taken as the onset of the apex cardiographic upstroke) and the end of diastole (taken as mitral valve closure) (Fig. 2) in normal individuals, in patients with left bundle branch block, and in VDD pacemaker patients with an atrioventricular interval of $50 \mathrm{~ms}$. The regression equation describing this relation for these patients with increasing delay of left ventricular depolarisation was $\mathrm{y}=0.9565 \mathrm{x}+16 ; \mathrm{r}=0.93$.

Effects of increasing atrioventricular intervals on the end of diastole and onset of systole in paced patients Mitral valve closure occurred significantly earlier as the programmed atrioventricular interval was 
$\mathrm{AV}=50 \mathrm{~ms}$
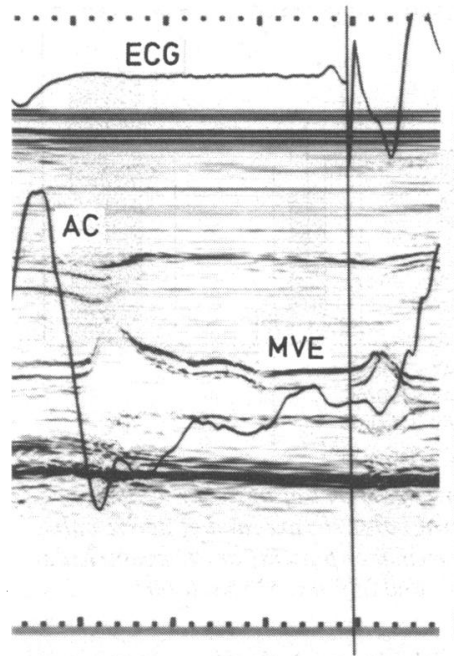

$A V=150 \mathrm{~ms}$

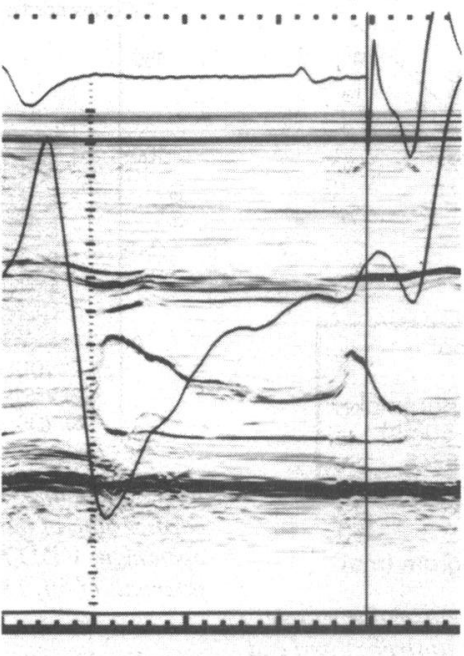

$\mathrm{AV}=250 \mathrm{~ms}$

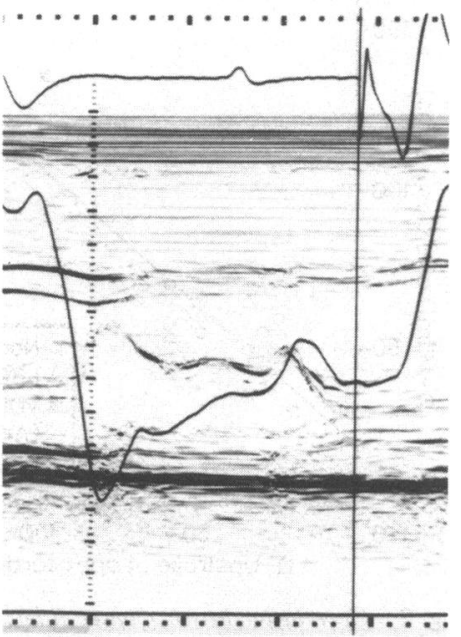

Fig. 4 An M mode echocardiogram of the mitral valve and a simultaneous apex cardiogram during VDD stimulation with atrioventricular intervals of 50,150, and $250 \mathrm{~ms}$. Early mitral valve closure with respect to the pacemaker spike (marked by the vertical line) is present at an atrioventricular interval of $250 \mathrm{~ms}$.

increased (Fig. 3). This is shown in an original recording from a patient with a low heart rate (Fig. 4) and at a faster heart rate even more pronounced changes were found (Fig. 5). As the atrioventricular interval was increased so the scatter of the individual values increased. This is shown by the rising standard deviation in Fig. 3.

In contrast, alterations in the atrioventricular interval had no effect on the timing of the upstroke of the apex cardiogram (mechanical systole), aortic valve opening (the onset of left ventricular ejection) (Fig. 3), or mitral valve opening (the onset of diastole) (Fig. 6).

\section{Left ventricular filling time}

Figure 6 shows the sequence of timing of mitral valve movements in all three groups with reference to the $Q$ waves or pacing spikes and also shows cycle
$\mathrm{AV}=50 \mathrm{~ms}$

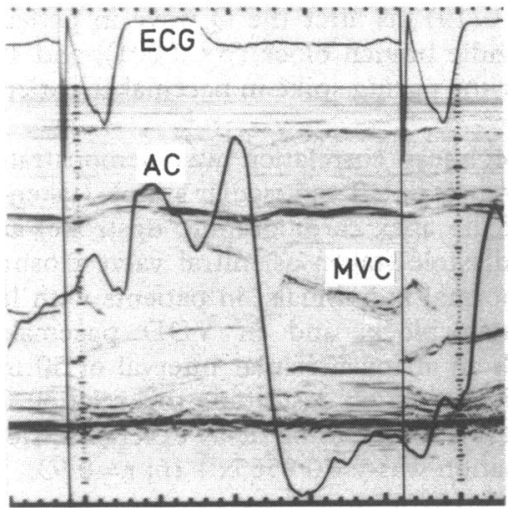

$\mathrm{AV}=150 \mathrm{~ms}$

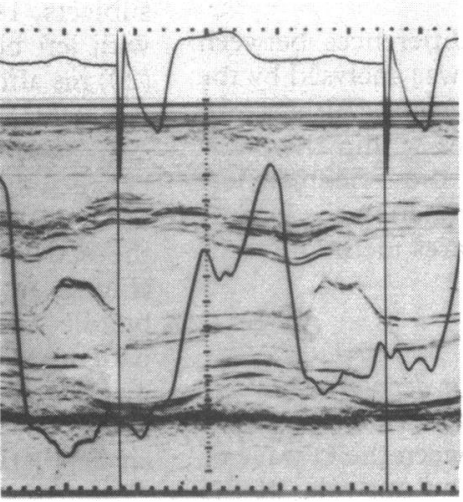

$\mathrm{AV}=250 \mathrm{~ms}$

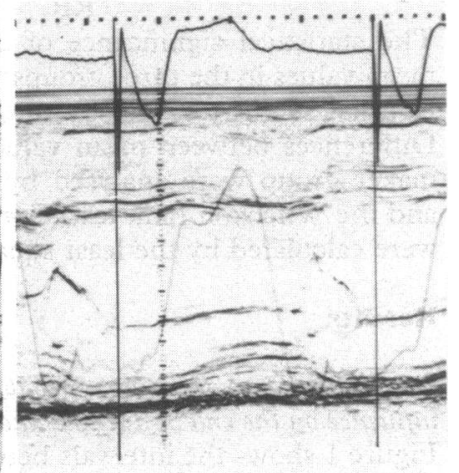

Fig. $5 M$ mode mitral valve echocardiograms and apex cardiograms during VDD stimulation with atrioventricular intervals of 50,150, and $250 \mathrm{~ms}$ in a patient with short cycle length. The mitral $A$ wave is diminished at $A V 150$ ms and leaflets close early. The mitral $A$ wave is not visible at $A V 250$ ms and leaflets close before the pacemaker spike, causing considerable reduction of left ventricular filling time. 


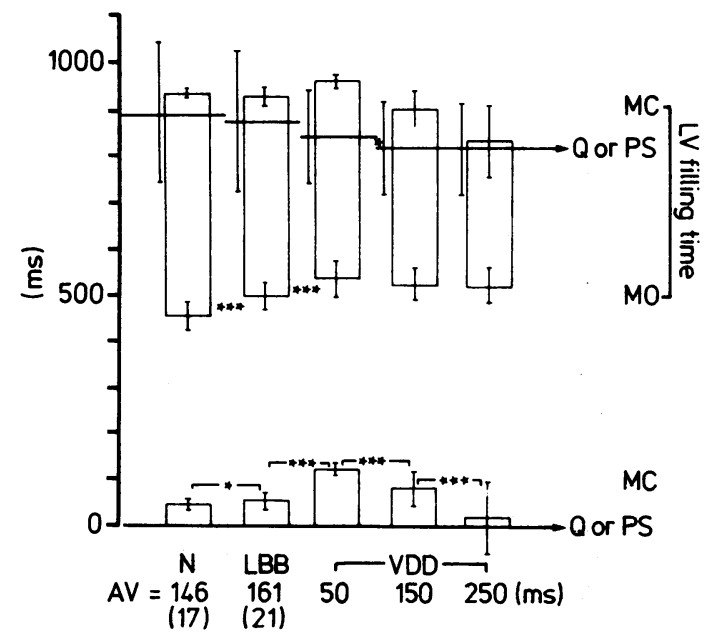

Fig. 6 Timing (mean (SD)) of mitral valve movements (MC, closure; MO, opening) throughout the cardiac cycle (with the $Q$ wave or pacemaker spike (PS) as reference points) in the group of normal individuals $(N)$, in patients with left bundle branch block ( $L B B$ ), and in patients with $V D D$ pacemakers set at atrioventricular intervals of 50,150, and $250 \mathrm{~ms}$.

length and the duration of left ventricular filling time. Mean cycle length was non-significantly different in controls, patients with left bundle branch block, and patients with pacemakers set with an atrioventricular interval of $50 \mathrm{~ms}$. With increasing atrioventricular delay there was a small but significant decrease in cycle length (Fig. 6).

In the pacemaker patients left ventricular filling time significantly decreased with increasing atrioventricular intervals. It was $441(94) \mathrm{ms}$ at the atrioventricular interval of $50 \mathrm{~ms}, 372(104) \mathrm{ms}(\mathrm{p}<0.03)$ when the interval was $150 \mathrm{~ms}$, and 304 (103) ms $(\mathrm{p}<0.02$ compared with a setting of $150 \mathrm{~ms}$ ) when the interval was $250 \mathrm{~ms}$. This reduction in left ventricular filling time was due to alterations in the timing of mitral valve closure since the timing of mitral valve opening was unchanged (Fig. 6).

Normalised left ventricular filling time was $\mathbf{5 3}$ (6) $\%$ in control subjects, somewhat less in patients with left bundle branch block (48 (7)\%, $\mathrm{p}<0.025)$, and did not differ from normal in pacemaker patients with an atrioventricular interval of $50 \mathrm{~ms}$ $(50(6) \%)$. With increasing atrioventricular intervals there was a significant reduction to 45 (9)\% $(p<0.007)$ when the atrioventricular delay was 150 $\mathrm{ms}$, and to $38(10) \%(\mathrm{p}<0.02$ compared with an interval of $150 \mathrm{~ms}$ ) when the atrioventricular delay was $250 \mathrm{~ms}$; both these values were significantly less than normal $(\mathrm{p}<0.004$ and $\mathrm{p}<0.001)$.

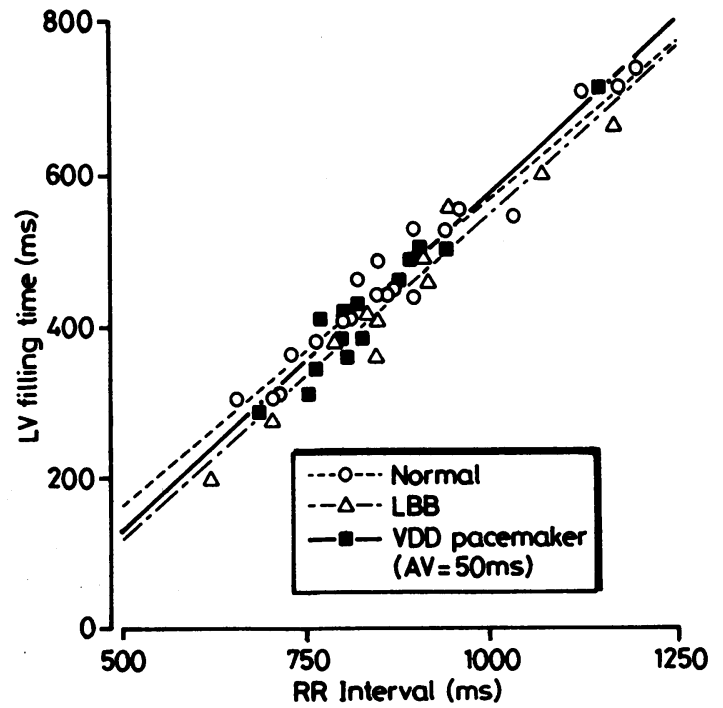

Fig. 7 Correlation between left ventricular filling time and cycle length in the group of normal individuals, in patients with left bundle branch block ( $L B B$ ), and in VDD pacemaker patients. The regression lines are given for each group.

The well known correlation between filling time and cycle length is demonstrated in Fig. 7 for normal individuals $(r=0.97)$, patients with left bundle branch block $(r=0.98)$, and VDD pacemaker patients at an atrioventricular interval of $50 \mathrm{~ms}$ $(r=0.93)$. Clearly these regression lines are not different from each other. The equation for all indi-

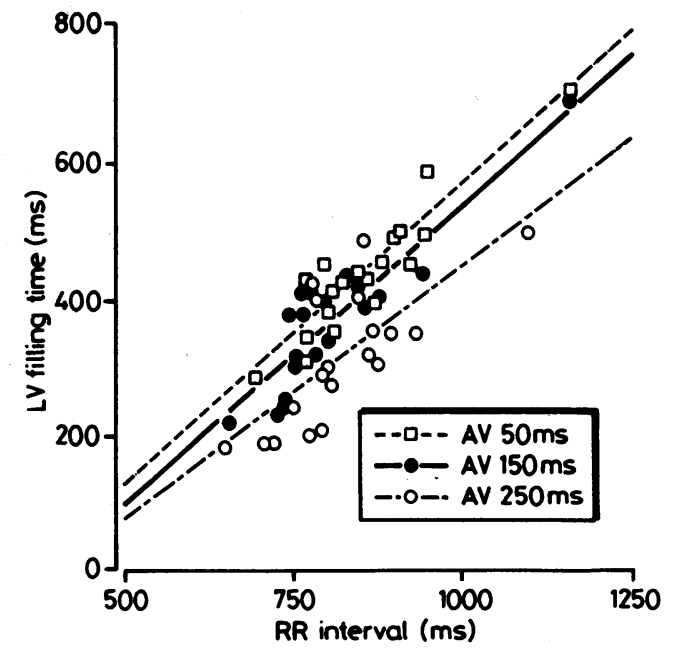

Fig. 8 Correlation between left ventricular filling time and cycle length in 19 VDD pacemaker patients at atrioventricular intervals of 50,150, and $250 \mathrm{~ms}$. 
viduals was $y=0.857 x-292(r=0.96)$. With longer atrioventricular intervals, however (Fig. 8), the line was shifted downwards. The equations were $y=0.909 x-334$ for an atrioventricular delay of 50 $\mathrm{ms}, \mathrm{y}=0.892 \mathrm{x}-350$ at a delay of $150 \mathrm{~ms}$, and $y=0.767 x-311$ when the delay was $250 \mathrm{~ms}$; the final value is significantly different from that seen with an interval of $50 \mathrm{~ms}(\mathrm{p}<0.001)$.

\section{Mitral $A$ wave}

The onset of the mitral A wave, signifying the mechanical effect of atrial contraction, occurred 81 (23) $\mathrm{ms}$ before the next $\mathrm{Q}$ wave in normal controls, 86(28) $\mathrm{ms}$ before the next $Q$ wave in patients with left bundle branch block, and only 5 (17) $\mathrm{ms}$ before the pacing spike in pacemaker patients in whom the atrioventricular interval was set at $50 \mathrm{~ms}(p<0.001$ compared with both controls and left bundle branch block patients). As the atrioventricular interval was increased in the pacemaker patients the onset of the mitral A wave occurred, as expected, at increasingly longer intervals before the pacing spike. These intervals were $76(24) \mathrm{ms}(\mathrm{p}<0.01)$ when the atrioventricular delay was $150 \mathrm{~ms}$ and 148 (38) ms ( $<<0.001$ ) when the delay was $250 \mathrm{~ms}$. As the mitral A wave occurred longer before the pacing spike it began to exert an effect on mitral closure not seen with shorter atrioventricular delays when mitral closure occurs as a result of ventricular systole. This resulted in the correlation between onset of left ventricular systole and mitral valve closure being lost when the atrioventricular delay was long.

\section{Discussion}

Because of the short time available in the cardiac cycle and the large number of events occurring during it, considerable synchronisation between myocardial and valve movement is necessary if the heart is to function efficiently. The study of time intervals in the cardiac cycle has a long history. ${ }^{8}$ It has mainly concentrated on systolic events, despite the fact that asynchrony during diastole is of great importance since any impairment in ventricular filling must result in an equivalent impairment or ejection.

In 1921 Wiggers defined the onset of systole as coinciding with the closure of the atrioventricular valves. ${ }^{9}$ Subsequent use of phono- and echocardiography has shown many exceptions to this definition. Considerable variation in the timing of mitral valve closure, which means that it does not necessarily coincide with the onset of mechanical systole, has been described in the presence of raised left atrial pressure, ${ }^{1011}$ intraventricular conduction disturbances, ${ }^{1-3}$ atrioventricular block, ${ }^{4-6}$ and atrial fibrillation. ${ }^{12}$ Since mitral valve closure must be the end of left ventricular filling its relation to the onset of systole is of considerable importance.

The study of patients with dual chamber pacemakers is of great interest. Firstly, these patients represent a unique physiological model in which the timing of cardiac events may be studied. The interval between the normal $P$ wave and the ventricular pacing stimulus which it triggers when the pacemaker is in the VDD mode can be altered by reprogramming the pacemaker in the absence of any other physiological change. Therefore the effect of atrial and ventricular contraction on mitral valve closure and its relation to the onset of mechanical systole can be studied over a wide range of atrioventricular intervals. Secondly, study of these patients provides practical information about the atrioventricular interval that allows the longest diastolic filling period and thus the most efficient cardiac function.

\section{Intraventricular conduction delay and the onset of systole}

The electromechanical delay, detected as the interval between the $Q$ wave and the onset of the upstroke of the apex cardiogram, which coincides with the onset of left ventricular pressure rise, ${ }^{7}$ was about 50 ms. This accords with previous reports. ${ }^{13}$ In the paced patients we studied, this electromechanical delay lasted considerably longer (about $120 \mathrm{~ms}$ ) and was the same for all three atrioventricular intervals. This delay may be partly because of slow initial spread of depolarisation from the right ventricular pacing site until it gains access to the faster specialised conducting system. If this is the case some increase in the electromechanical delay might be expected in the group of patients with left bundle branch block. This was not found by apex cardiography but other indicators suggest that a small delay does occur. Firstly, mitral valve closure was slightly delayed and is, as will be clear later, dependent on the onset of systole in these patients. Secondly, aortic valve opening, denoting the onset of left ventricular ejection was also delayed compared with values in normal individuals. Others have found a considerable delay of right ventricular contraction in right bundle branch block but they stated left ventricular contraction is not delayed in left bundle branch block because they claimed to have demonstrated normal timing of mitral valve closure. $^{23}$

Their point of reference, however, was closure of the tricuspid valve. When we reanalysed their results with reference to the electrocardiographic $Q$ wave we found a delay in mitral valve closure that is similar to that observed in the present study.

Despite the differences in the intervals between 
electrical and mechanical events in these three groups of patients (that is normal controls, patients with left bundle branch block, and patients with VDD pacemakers with an atrioventricular interval of $50 \mathrm{~ms}$ ) the normal sequence of left ventricular pressure rise and mitral valve closure was maintained in all three groups. All three groups showed the same strong linear relation between the onset of systole and mitral valve closure. This clearly demonstrates the predominant effect of the onset of systole in closing the mitral valve and thus ending diastole in all three groups despite the increasing delay in the onset of systole due to impaired conduction in two of the groups.

The phases of the cardiac cycle are often defined with reference to the electrocardiogram with end diastole being taken as coinciding with the $Q$ wave or pacing spike. By this definition the period of up to $127 \mathrm{~ms}$ after the pacing spike would be assigned to systole, despite the fact that the mitral valve is still open. In theory this period is long enough to permit all of rapid left ventricular filling ${ }^{14} 15$ although in practice it is more commonly used for left ventricular filling due to atrial contraction. Similarly such traditional definitions of end diastole may cause considerable inaccuracy in the assessment of end diastolic left ventricular dimension by echocardiographic or scintigraphic techniques since some or all of the atrial contribution to ventricular filling will be neglected because it occurs after the $Q$ wave. To avoid these problems we regard end diastole as occurring at the moment when the mitral valve closes, and the onset of systole as the moment when left ventricular pressure begins to rise.

\section{Effect of various atrioventricular intervals on the end of diastole}

Each increase in the atrioventricular interval by 100 $\mathrm{ms}$ in the VDD paced patients made the coaptation of the mitral leaflets in relation to the pacing spike occur $50 \mathrm{~ms}$ earlier. This cannot be due to the mitral valve being closed earlier by left ventricular contraction, since the timing of the onset of systole in relation to the pacing spike did not change. The most likely explanation is that this was the result of a change in the mechanism causing mitral valve closure as the atrioventricular delay was increased. When the delay is short, closure is effected by ventricular systole, and this mechanism accords with the strong linear relation between the timing of the two events and the delay of $50 \mathrm{~ms}$. At longer atrioventricular intervals atrial contraction may become the most important determinant of mitral closure. This concept is supported by echocardiographic studies of mitral closure in patients with complete heart block ${ }^{4-6}$ in whom wide variations in the inter- val between atrial and ventricular contraction can be studied. It is suggested that atrial contraction raises left ventricular pressure so that as atrial systole ends there is a reverse pressure gradient which closes the valve unless ventricular systole intervenes to close it first. Therefore the mechanism of closure will vary depending on the atrioventricular interval.

In sinus rhythm a PR interval of more than $\mathbf{2 0 0}$ $\mathrm{ms}$ is required before mitral valve closure precedes ventricular contraction (that is as a result of atrial contraction). ${ }^{16}$ The situation is different in patients with ventricular pacing. Movement of the valve at various atrioventricular intervals was studied by cinefluorography in a patient with a Starr-Edwards mitral prosthesis, complete heart block, and a ventricular pacemaker. ${ }^{17}$ This showed that valve closure preceding ventricular systole occurred at much shorter atrioventricular intervals $(>100 \mathrm{~ms}$ ). Our study also shows that this effect often occurs when the interval is set at $150 \mathrm{~ms}$. This difference between the interval required for atrial contraction to produce mitral closure in paced patients and those required in sinus rhythm is due to the longer delay in the onset of systole with reference to the electrocardiogram (pacing spike and $Q$ wave respectively) in paced patients. This additional delay, which is approximately $80 \mathrm{~ms}$, means that the functional atrioventricular interval lasts $80 \mathrm{~ms}$ longer in paced patients than it does in patients in sinus rhythm with the same electrocardiographic PR interval. Therefore, theoretically, atrioventricular delay settings of 50-100 ms should be sufficient to maintain the normal intervals between atrial and ventricular contraction in paced patients.

These observations are of considerable practical importance. An atrioventricular interval of $150 \mathrm{~ms}$ is commonly used when programming VDD pacemakers because this is erroneously thought to correspond to the functional interval in sinus rhythm. Our study, however, shows that at this setting mitral valve closure frequently occurred before the onset of ventricular systole, and thus late diastole was not available for ventricular filling. It is also commonly thought that short atrioventricular intervals lead to the atrial A wave being cut off prematurely by ventricular systole. This study shows that this is not the case. At a setting of $50 \mathrm{~ms}$ the onset of the mitral A wave occurred 5 (17) $\mathrm{ms}$ before the pacing spike. The delay of ventricular activation until $127 \mathrm{~ms}$ after the pacing spike allowed sufficient time for a mitral A wave of normal duration $(132 \mathrm{~ms})$ to occur at this setting. This value for the duration of the $A$ wave is almost identical with that obtained from simultaneous left ventricular and left atrial tracings in patients with both normal and abnormal left ventricular function. ${ }^{18}$ When the atrium and ventricle are 
paced sequentially, the delay between the atrial pacing spike and atrial activation, which may vary between 30 and $120 \mathrm{~ms},{ }^{19}$ should be assessed individually and added to the proposed range of 50-100 ms advocated here for the VDD mode.

The reversed pressure gradient by which atrial contraction closes the mitral valve is determined by the force of atrial contraction. ${ }^{4}$ It may be counteracted if atrial contraction is weak, atrial pressure is high, or if blood flow is increased. These additional influences may explain the wide individual scatter in the timing of mitral valve closure at long atrioventricular intervals. There were four patients in whom the timing of leaflet coaptation was unchanged despite increasing intervals. One had poor left ventricular function due to previous anterior myocardial infarction, and there was radiographic and clinical evidence of raised left atrial pressure. The other three were physically fit youths in whom the mitral valve reopened after the $A$ wave. These four patients contribute to the exceptional individual values seen in Fig. 8.

Although in this study we examined only the sequence of haemodynamic events in the left side of the heart, there is no evidence that the sequence in the right heart is essentially different. It is well known that the right atrial $\mathbf{P}$ wave occurs about 30 $\mathrm{ms}$ before depolarisation of the left atrium. Phonocardiographic ${ }^{3}$ and invasive studies ${ }^{2}$ of patients with left bundle branch block or right ventricular pacemakers have shown that the onset of contraction of both ventricles is simultaneous. Thus the selection of the short atrioventricular interval, shown in this study to benefit left ventricular filling, is unlikely to affect right ventricular function in an adverse way.

\section{Left ventricular filling time}

Left ventricular filling time was significantly shortened in our patients with VDD pacemakers when the atrioventricular delay was set at $150 \mathrm{~ms}$ or longer. When the delay was $150 \mathrm{~ms}$ filling time was reduced by $60 \mathrm{~ms}$ and this reduction increased to $130 \mathrm{~ms}$ when the delay was set at $250 \mathrm{~ms}$. Timing of mitral valve opening was the same at all atrioventricular intervals. With an atrioventricular delay of $150 \mathrm{~ms}$ left ventricular filling time was reduced by a combination of reduced cycle length and earlier mitral valve closure. With an increase of the programmed delay to $250 \mathrm{~ms}$ the further reduction in filling time was entirely due to even earlier mitral valve closure since the cycle length remained constant. The resulting interval between mitral valve closure and the onset of left ventricular systole of up to $100 \mathrm{~ms}$ serves neither left ventricular filling nor ejection.
This waste of time may be harmless at a normal heart rate and with a normal left ventricle because up to $80 \%$ of the left ventricular filling occurs during the initial rapid filling phase of diastole which lasts about $120 \mathrm{~ms}$ and the remaining $20 \%$ can easily be completed during diastasis and with atrial contraction. Elderly pacemaker patients, however, may have other cardiac abnormalities such as ischaemic heart disease or left ventricular hypertrophy that lead to disturbed left ventricular diastolic function. Seven of our 19 patients with VDD pacemakers had evidence of such abnormality with delayed mitral valve opening consistent with left ventricular relaxation abnormalities. In these circumstances the amount of left ventricular inflow during the early rapid filling period is reduced to $60 \%$ or less and thus the time available for left ventricular filling in late diastole becomes far more important and may determine the left ventricular end diastolic volume and stroke volume.

The wasted time in late diastole with long atrioventricular intervals also becomes important during exercise, particularly in the presence of abnormal left ventricular diastolic function when the heart rate increases and left ventricular filling time is reduced. Since left ventricular filling time is strongly dependent on cycle length, we analysed this relation in all groups (Fig. 7). The regression equations were identical for the normal controls, patients with left bundle branch block, and VDD pacemaker patients with an atrioventricular interval of $50 \mathrm{~ms}$. These equations show that theoretically left ventricular filling time would be zero at mean cycle length of $320 \mathrm{~ms}$. The relation between cycle length and filling time was shifted downwards as atrioventricular intervals increased. Thus with an atrioventricular interval of $250 \mathrm{~ms}$ the cycle length producing a theoretical filling time of zero increases to $405 \mathrm{~ms}$. Therefore longer atrioventricular intervals have an increasingly greater negative influence on filling time as heart rate increases. We conclude that a short atrioventricular interval of 50 or $75 \mathrm{~ms}$ in patients with VDD pacemakers is likely to promote more physiological conditions by maximising left ventricular filling time both at rest and on exercise. This is likely to be of particular benefit in the groups of patients who most need this type of pacemaker; the young who require high cardiac outputs at high heart rates and patients with impaired left ventricular diastolic function.

\section{References}

1 Wolferth CC, Margolies A. Asynchronism in contraction of ventricles in so called common type of bundle-branch block: its bearing on determination of 
side of significant lesion and on mechanism of split first and second heart sounds.Am Heart $\mathcal{f}$ 1935; 10: 425-38.

2 Braunwald E, Morrow AG. Sequence of ventricular contraction in human bundle branch block. Am $\mathfrak{f}$ Med 1957; 23: 205-11.

3 Haber E, Leatham A. Splitting of heart sounds from ventricular asynchrony in bundle-branch block, ventricular ectopic beats, and artificial pacing. Br Heart $\mathcal{f}$ 1965; 27: 691-6.

4 Zaki A, Steinmetz E, Feigenbaum H. Role of atrium in closure of mitral valve in man. Am $\mathcal{F}$ Physiol 1969; 217: 1652-9.

5 Shah PM, Dramer DH, Gramiak R. Influence of the timing of atrial systole on mitral valve closure and on the first heart sound in man. Am $\mathcal{F}$ Cardiol 1970; 26: 231-7.

6 Burggraf GW, Craige E. The first heart sound in complete heart block: phono-echocardiographic correlations. Circulation 1974; 50: 17-24.

7 Venco A, Gibson DG, Brown DJ. Relation between apexcardiogram and changes in left ventricular pressure and dimension. Br Heart $\mathcal{f}$ 1977; 39: 117-25.

8 Lewis RP, Rittgers SE, Forester WF, Boudoulas H. A critical review of the systolic time intervals. Circulation 1977; 56: 146-58.

9 Wiggers CJ. Studies on the consecutive phases of the cardiac cycle. I. The duration of the consecutive phases of the cardiac cycle and the criteria for their precise determination. Am $\mathcal{F}$ Physiol 1921; 56: 415-38.

10 Wells B. The assessment of mitral stenosis by phonocardiography. Br Heart $\mathcal{f} 1954$; 16: 261-6.
11 Blömer H, Rudolph W. Klinische Kriterien zur Erfassung der Höhe des linken Vorhofdruckes bei Mitralstenosen. Z Kreislaufforschung 1959; 48: 350-62.

12 Cieslinski A, Hui WKK, Oldershaw PJ, Gregoratos G, Gibson D. Interaction between systolic and diastolic time intervals in atrial fibrillation. Br Heart $\mathcal{F} 1984$; 51 : 431-7.

13 Harris WS. Systolic time intervals in the noninvasive assessment of left ventricular performance in man. In: Mirsky J, Ghista DN, Sandler H, eds. Cardiac mechanics: physiological, clinical and mathematical considerations. New York and London: John Wiley, 1974: 233-92.

14 Gibson DG, Brown D. Measurement of instantaneous left ventricular dimension and filling rate in man, using echocardiography. Br Heart $\mathcal{f}$ 1973; 35: 1141-9.

15 Hui WKK, Gibson DG. Mechanisms of reduced left ventricular filling rate in coronary artery disease. $\mathrm{Br}$ Heart f 1983; 50: 362-71.

16 Craige E. On the genesis of heart sounds. Contributions made by echocardiographic studies. Circulation 1976; 53: 207-9.

17 Hamby RJ, Aintablian A, Wisoff BG. The role of atrial systole in valve closure. Chest 1973; 64: 197-202.

18 Braunwald E, Frahm C. Studies on Starling's law of the heart IV. Observations on the hemodynamic functions of the left atrium in man. Circulation 1961; 24: 633-42.

19 Alt E, Wirtzfeld A, Seidl K, Haller F, von Bibra H, Sauer E. Delayed atrial excitation following bifocal pacemaker stimulation. $Z$ Kardiol 1983; 72: 245-8. 